\title{
Frequency ratio determines discrimination of concentric radial frequency patterns in the peripheral visual field
}

\author{
Yang Feng ${ }^{1,2} \cdot$ Qiong $\mathrm{Wu}^{1,3} \cdot$ Jiajia Yang $^{3} \cdot$ Satoshi Takahashi ${ }^{3} \cdot$ Yoshimichi Ejima $^{3} \cdot$ Jinglong $\mathrm{Wu}^{3,4} \cdot$ Ming Zhang $^{1,3,5}$
}

Published online: 4 September 2020

(C) The Psychonomic Society, Inc. 2020

\begin{abstract}
Using a radial frequency discrimination task that has not been tested in many previous studies, we examined the dependence of the pattern radius (4 to $16 \mathrm{deg}$ ) on the radial frequency thresholds of two different types of concentric radial frequency (RF) patterns: constant circular contour frequency (CCF) RF patterns with different radii, which have the constant physical length of modulation cycle in external real-world space, and constant radial frequency magnified RF patterns with different radii, which have the constant cortical length of modulation cycles. These two types RF patterns used as the reference stimuli had an equal maximum orientation difference from circularity regardless of change in radius. The discrimination threshold expressed by the frequency ratio between RF patterns of different frequencies vs. radius functions for the constant CCF RF patterns indicated different functional forms dependent on the modulation amplitude of the RF patterns. The thresholds increased with increasing pattern radius for small modulation amplitude RF patterns but were relatively flattened for large-amplitude RF patterns. This dependence was ascribed to the eccentricity effect wherein the deformation thresholds for discriminating the RF pattern from a circle increase with increasing stimulus eccentricity (Feng et al. 2020). The discrimination thresholds vs. radius functions for the magnified RF patterns were also flattened for different modulation amplitudes and frequencies. The thresholds (frequency ratio) were similar at all eccentricities. Cortical magnification neutralized the eccentricity effect observed for the constant CCF patterns.
\end{abstract}

Keywords Concentric radial frequency pattern · Eccentricity effect · Discriminability · Frequency ratio · Orientation ratio · Retinocortical mapping $\cdot$ Discrimination task

\section{Introduction}

Radial frequency (RF) patterns, which were introduced by Wilkinson, Wilson, and Habak (1998), have frequently been

Yang Feng and Qiong Wu were co-first authors

Qiong $\mathrm{Wu}$

wuqiong@usts.edu.cn

$\triangle$ Ming Zhang

psyzm@suda.edu.cn

1 Department of Psychology, Suzhou University of Science and Technology, Suzhou, China

2 Biomedical Engineering Laboratory, Graduate School of Natural Science and Technology, Okayama University, Okayama, Japan

3 Cognitive Neuroscience Lab. Graduate School of Interdisciplinary Science and Engineering in Health Systems, Okayama University, 3-1-1 Tsushima-naka, Okayama, Japan

4 Beijing Institute of Technology, Beijing, China

5 Department of Psychology, Soochow University, Suzhou, China used to investigate aspects of shape processing. The simple mathematical definition of RF patterns has made RF patterns a popular stimulus in psychophysical, physiological, and imaging studies (Wilkinson et al., 2000; Loffler, 2008; Loffler, 2015; Salmela et al., 2016). However, Wilkinson et al. (1998) explained the mathematical limitation of RF patterns and stressed their differences from Fourier shape descriptors, which can in principle be used to create any kind of closed two-dimensional shapes. Recently, Schmidtmann and Fruend (2019) demonstrated that compound RF patterns can represent only a very small subset of natural and synthetic shapes, but the RF patterns are not suited as universal shape descriptors utilized by the visual system. Schmidtmann and Fruend (2019) also noted that it is important to keep in mind that studies of RF patterns implicitly restrict them to only certain ranges of perceptually meaningful dimensions. Despite this limitation, there remains the possibility that accumulating structural properties of the visual performance of RF patterns further by using a wider range of stimulus condition and a different visual task and by exploring a possible model may open a new perspective and lead to deep insight into the shape encoding mechanism. 
Here, we provide an additional test on the discrimination performance of RF patterns presented in the peripheral visual field. We asked three questions regarding the visual performance to concentric RF patterns with a radius that varied from 4 to $16 \mathrm{deg}$, using a frequency discrimination task: What are the most important factors to determine the discrimination between different RF patterns? How does the discrimination performance vary with eccentricity? How can spatial scaling (the retinocortical transformation) account for the eccentric properties of the discrimination performance?

RF patterns are constructed by applying a radial sinusoidal modulation to the radius $r_{0}$ such that the radius of the deformed pattern at polar angle $\theta$ in radians is:

$\mathrm{r}=r_{0}(1+A \cos (\omega \theta+\varphi))$

where $r_{0}$ is the mean radius, $A$ is the radial modulation amplitude, and $\omega$ is the radial frequency. The primary parameters of visual performance for RF patterns are modulation amplitude (A) on the radial coordinate and radial frequency $(\omega)$ on the angular coordinate. These two parameters are in an orthogonal relationship with each other. They can be varied independently. However, they cannot affect the salience of the modulation independently. Properties such as local curvature and the maximum local orientation difference from circular vary with both of these parameters. Most previous studies have used a deformation threshold task in which the modulation amplitude threshold to discriminate the RF pattern from a circular pattern is measured by manipulating the modulation amplitude. In previous studies, the radial frequency of RF patterns was used as an important parameter that affects visual performance. Surprisingly, to our knowledge, radial frequency discrimination tasks, in which the discrimination threshold to detect the difference in the frequency between two RF patterns is measured by manipulating the radial frequency of the RF patterns, has rarely been studied; some exceptions, however, include psychophysical studies that have used radial frequency identification tasks (Wilkinson et al., 1998; Poirier and Wilson, 2006; Bell et al., 2007; Dickinson et al., 2013). The frequency discrimination threshold task used in this study may plausibly shed new light on the aspect of the visual performance of RF patterns. The radial frequency discrimination task may be useful to examine the sensitivity to changes in the angular coordinate of RF patterns and the frequency selectivity in the processing of RF patterns.

Achtman, Hess, and Wang (2000), reported that the performance of RF patterns presented in the peripheral retinal location decreased, and Achtman et al. (2000) Zolubakand GarciaSuarez $(2017,2018)$, and Zolubak, Schmidtmann and GarciaSuarez(2018) showed that the eccentricity effect could be neutralized by scaling with respect to cortical magnification factors. In these last studies, the researchers used a deformation task. In the present study, we tested the eccentricity effect for magnified and nonmagnified concentric presentations of RF patterns using the radial frequency discrimination task.

In the present study, using constant circular contour frequency $(\mathrm{CCF})$ patterns with different radii, which have the constant physical length of modulation cycle in external real-world space, and constant radial frequency magnified RF patterns with different radii, which have the constant cortical length of modulation cycles, we examined the effect of the RF pattern radius (between 4 and $16 \mathrm{deg}$ ) on radial frequency discrimination thresholds. As a consequence, we found that the discrimination thresholds between different frequency-magnified patterns were determined by the frequency ratio of the two patterns, regardless of different pattern radii and reference frequencies.

\section{General methods}

\section{Participants}

The participants included one of the authors and four healthy students (three males, $27.7 \pm 3.8$ years old). All participants had normal vision or corrected-to-normal vision and provided written informed consent for the procedure, which was previously approved by the ethics committee of Okayama University and was organized to ensure conformity to the Declaration of Helsinki.

\section{Apparatus}

MATLAB (MathWorks, Inc.) and Psychophysics Toolbox (Brainard, 1997), which provides high-level access to the Clanguage Video Toolbox (Pelli, 1997), were used to generate the custom stimuli used in this experiment. The program was run on a ThinkPad T540p notebook (Lenovo). Stimuli were presented on a SHARP-PN455 (SHARP) display with a 60$\mathrm{Hz}$ frame rate, 8-bit colors and $1920 \times 1080$ pixels. The gamma nonlinearity of the screen was corrected using a look-up table that was obtained via calibration through a KONICAMINOLTA CS-100A (Konica Minolta Japan, Inc.) spectroradiometer. The color of the stimuli was set to CIE $1931, x=0.34, y=0.33$, and the mean luminance of the screen was $175 \mathrm{~cd} / \mathrm{m}^{2}$. The stimulus luminance was modulated around a mean luminance of $175 \mathrm{~cd} / \mathrm{m}^{2}$ about each eccentricity, while the remainder of the screen was maintained at the mean luminance.

The participants viewed the display monocularly under dim room illumination at a distance of $80 \mathrm{~cm}$. Each display subtended $63.3 \mathrm{deg}$ horizontally and $38.2 \mathrm{deg}$ vertically. The participants were instructed to sit on a chair, put their chin on a chin-stand to ensure that the head did not move and maintained the distance from the screen, and keep their fixation at the fixation point presented in 
the center of the display during the presentation of the target stimuli.

\section{Base stimuli}

The base pattern used in this study was a circular contour with a cross-sectional luminance profile defined by a radial fourth derivative of a Gaussian (D4). The pattern used in this study was the same as that used by Wilkinson et al. (1998).

The equation for the circle is

$\mathrm{D} 4(\mathrm{r})=\mathrm{C} \times \exp \left(-\frac{\left(\mathrm{r}-\mathrm{r}_{0}\right)^{2}}{\sigma^{2}}\right) \times\left(1-4 \frac{\left(\mathrm{r}-\mathrm{r}_{0}\right)^{2}}{\sigma^{2}}+\frac{4}{3} \frac{\left(\mathrm{r}-\mathrm{r}_{0}\right)^{4}}{\sigma^{4}}\right)$

where $\mathrm{C}$ is the pattern contrast, which was $100 \%$ through all current experiments; $r_{0}$ is the mean radius of the stimulus, the center of which was located at the fixation point; and $\sigma$ determines the thickness of the cross-sectional profile.

The base circle was deformed by applying a radial sinusoidal modulation to the radius $r_{0}$ in Eq. (2) such that the radius of the deformed pattern at polar angle $\theta$ in radians was as shown in Eq. (1).

The angular phase of the pattern is determined by $\varphi$. The radial modulation amplitude (A) was not permitted to exceed 1.0 , and the radial frequency was always an integer value.

\section{Peripheral sensitivity for radial frequency discrimination}

Schmidtmann, Logan, Keendy, Gordon, and Loffler (2015) tested the hypothesis of enhanced sensitivity to a range of stimuli within the lower visual field by measuring discrimination sensitivity for orientation, curvature, shape (radial frequency patterns), and faces at various para-central locations and eccentricities. Peripheral sensitivity was isotropic for orientation and curvature. By contrast, observers were significantly better at discriminating shapes throughout the lower visual field compared to elsewhere. For faces, peak sensitivity was found in the lefty visual field. Note here that the visual field preference was observed for small visual stimuli. To our knowledge, no study for large RF stimuli such as our experimental stimuli (8 32-diameter stimuli) has been examined. No one knows whether the low field preference is also observed or not for our large RF stimuli. This problem is interesting, but beyond the scope of the present study.

Our radial frequency discrimination which is closely related to orientation or curvature discrimination is isotropic in sensitivity in the peripheral visual field.

\section{Procedure}

The thresholds to discriminate the target radial frequency from the reference frequency were measured using a three-alternative forced-choice procedure and the method of constant stimulus. In the frequency discrimination experiments, there were seven radial frequencies for the target stimuli with constant modulation amplitude: one frequency was the same as the reference frequency (for example 9 cycles $/ 360 \mathrm{deg}$ ), three frequencies were higher $(10,11$, and 13 cycles $/ 360 \mathrm{deg}$ for the reference frequency of 9 cycles $/ 360 \mathrm{deg})$, and three frequencies were lower $(6$, 7 , and 8 cycles $/ 360 \mathrm{deg}$ ). They were arranged at nearly equal intervals on a logarithmic scale. When the target and reference have the same radial frequency, the participants may feel substantial difficulty in making a binary decision, e.g., "target has a higher radial frequency than the reference", or "target has a lower radial frequency than the reference". To circumvent this problem, we used the three-alternative forced-choice procedure rather than the two-alternative forced-choice procedure used by the majority of previous RF pattern research, in which the participants may select a third choice "target and reference have the same radial frequency". Additionally, we used the three-alternative forced-choice procedure rather than the two forced-choice procedure because the latter is known to present with a potential difficulty: at low signal intensities (small differences in frequency between target and reference), the participant (particularly a naïve observer) is often truly uncertain about the correct answer from a two-alternative forced-choice task. Being forced to make a choice even when one does not have the slightest idea regarding which response is correct may introduce an uncomfortable aspect to this task. This may be particularly important in situations in which the participant is confronted for the first time with this task or where it is infeasible to dispel the reluctance of the participants to act randomly because it would require too much explanation and might reduce the confidence of the participants in the seriousness of the investigation (Kaernbach, 2001). The feasibility of using a "guess-button" in addition to the conventional response keys defined in 2FAC tasks has also been proved to yield a more precise result (GarcíaPérez, 2010).

Participants were presented with a 5-min dark adaptation to the dim room and a 3-min light adaptation to the mean luminance of the display. This procedure was performed to acclimate the participant's eyes to quickly adapt to the mean luminance of the experimental display. Each trial subsequently consisted of two 200-ms stimulus presentation intervals with a $300-\mathrm{ms}$ ISI and a response interval; one presentation interval contained the reference stimulus, while the other presentation interval contained one of the target stimuli. The presentation order of the reference and target were randomized within a session. The phase of the stimuli was varied randomly between trials. This random-phase setting rendered it impossible 
for the subjects to predict the exact locations at which the maximum and minimum radial deviations would occur. The participants were instructed to indicate whether, compared to the first stimulus (S1), the frequency of the second stimulus (S2) was higher, lower or same using one of three buttons: one button for the S2 frequency being higher than the S1 frequency, another button for the S2 frequency being lower than the S1 frequency, and a third button for S2 and S1 having the same frequency (Fig. 1). With the participant's self-paced starting signal, the next session began. Within a session, the seven radial frequency targets were randomly presented, with each presented ten times, while the other stimulus parameters of the target and the reference were held constant. The four stimulus radii were randomly presented across sessions.

\section{Analysis}

We used a three-alternative forced-choice procedure. The responses of the participants were composed of "higher", "lower" and "same" responses. Based on the "higher" and "lower" responses, we obtained the following logistic curve equation:

$\mathrm{y}=\left(1+e^{\alpha+\beta x}\right)^{-1}$

where the responses of the participants are expressed from 0 to $100 \%$ on the ordinate. The abscissa corresponds to the target radial frequency from 6 cycles $/ 360$ deg to 13 cycles $/ 360 \mathrm{deg}$, and 9 cycles/360 deg is the reference radial frequency $\left(\omega_{R}\right)$. From the logistic curve, we obtained two key points on the abscissa: $\omega_{\mathrm{TH}}$, the frequency where the participants achieved $75 \%$ on the ordinate, and $\omega_{\mathrm{TL}}$, the frequency where the participants achieved $25 \%$ on the ordinate, as shown in Fig. 2.

We calculated the radial frequency discrimination thresholds by using the following equation:

Discrimination thresholds $=\frac{\ln \left(\omega_{T H}\right)-\ln \left(\omega_{T L}\right)}{2}$

$$
=\ln \left(\left(\frac{\omega_{T H}}{\omega_{T L}}\right)^{\frac{1}{2}}\right)
$$

Dickinson, McGinty, Webster, and Badcock (2012) showed that the quantity that was equated at thresholds for detection of modulation on RF patterns of different frequencies but with the same number of cycles of modulation was the maximum angular deviation from circularity. This was for patterns with relatively low frequencies of modulation that have been shown to exhibit integration of information across cycles, but not for patterns with higher frequency. It was consistent with the results of other study (Loffler et al., 2003). It is suggested that an important cue for the discrimination between RF different stimuli, regardless of information integration, may be the maximum angular deviation from circularity.

The maximum angular deviation (orientation difference) between an RF and a circle occurs where an RF and a circle intersect each other and given by the following equation (Dickinson et al., 2012; Schmidtmann and Kingdom, 2017).

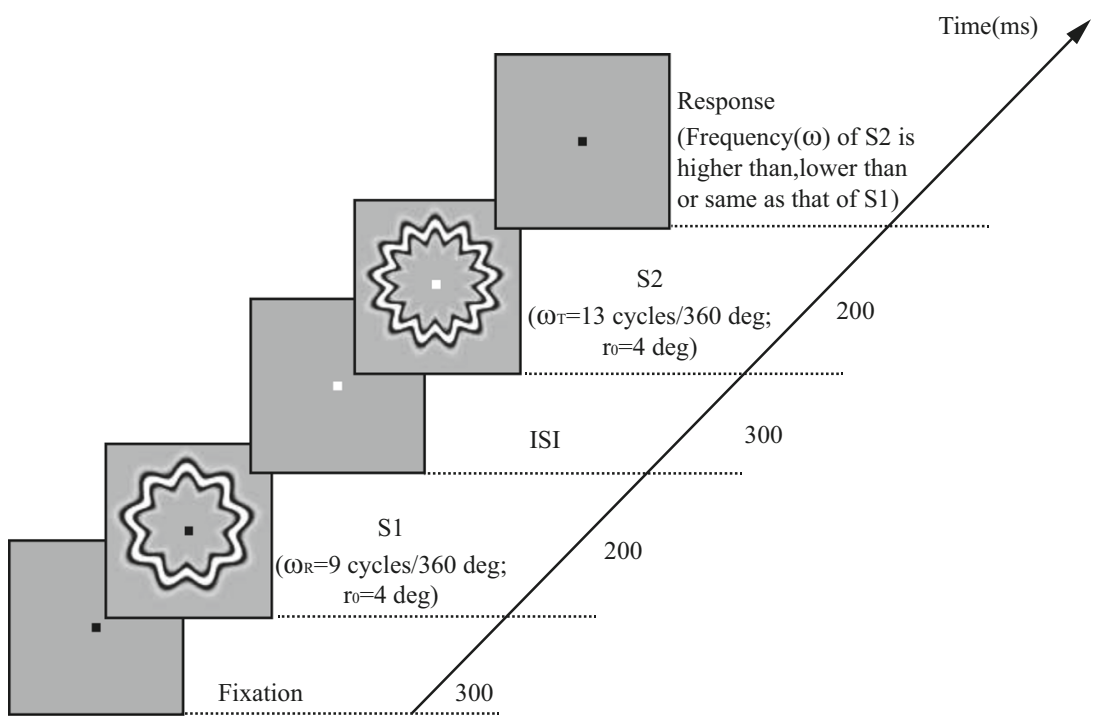

Fig. 1 Time course of the radial frequency discrimination experiment. Each trial consisted of two 200-ms stimulus presentation intervals with a 300-ms ISI and a response interval; one presentation interval contained a reference stimulus, while the other presentation interval contained a target stimulus. The participants were instructed to indicate whether, compared to the first stimulus (S1), the frequency $(\omega)$ of the second stimulus (S2) was higher, lower or the same using one of three buttons: one button for the S2 frequency being higher than the S1 frequency, another button for the $\mathrm{S} 2$ frequency being lower than the $\mathrm{S} 1$ frequency, and a third button for the S2 frequency being the same as the S1 frequency 
Relative Frequency of Responses $\left(\omega_{\mathrm{R}}=9 \mathrm{cycles} / 360 \mathrm{deg}\right)$

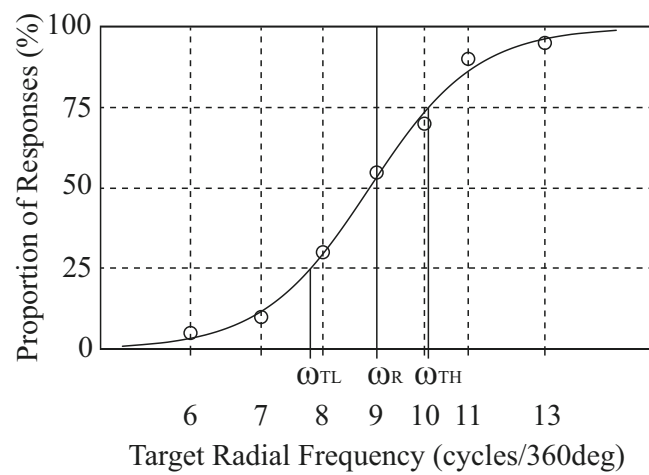

Fig. 2 Derivation of radial frequency discrimination thresholds. We used a three-alternative forced-choice procedure. The responses of the participants were composed of the "higher", "lower" and "same" responses. Based on the proportion of correct "higher" and "lower" responses versus the target radial frequency, we obtained a logistic curve equation, where the relative proportions of the "lower" and the "higher" responses are expressed from 0 to $100 \%$ on the ordinate. The abscissa indicates the target radial frequency from 6 to 13 cycles $/ 360 \mathrm{deg}$, and 9 cycles $/ 360$ deg is the reference radial frequency $\left(\omega_{R}\right)$. From the logistic curve, we obtained two key points on the abscissa, $\omega_{\mathrm{TH}}$, the frequency where the participants achieved $75 \%$ on the ordinate, and $\omega_{\mathrm{TL}}$, the frequency where the participants achieved $25 \%$ on the ordinate. We calculated the radial frequency discrimination thresholds by using the following equation: Discrimination threshold $=\frac{\ln \left(\omega_{T H}\right)-\ln \left(\omega_{T L}\right)}{2}=\ln \left(\left(\frac{\omega_{T H}}{\omega_{T L}}\right)^{\frac{1}{2}}\right)$

Maximum orientation differences $(\tan (\alpha))=A \omega$

Where $\alpha$ represents the angle between the tangential directions of an RF pattern and the circle at the crossing point. A and $\omega$ represent the modulation amplitude and the radial frequency of the RF pattern, respectively. The maximum orientation difference is abbreviated as MOD.

Thus, the ratio of MODs between two RF patterns of equal radius and modulation with $\omega_{1}$ and $\omega_{2}$ is equal to the radial frequency ratio of the two patterns, implying that the ratio of MODs may be a critical cue to discriminate two different RF patterns as well. The discrimination thresholds in the Eq. (4) is expressed also by the ratio of MODs of the two patterns.

\section{Experiment 1}

\section{Experimental stimuli: Constant circular contour frequency (CCF) RF pattern stimuli}

The CCF is defined by the number of radial cycles per deg of unmodulated contour length measured in deg of viewing angle. This is an important factor in determining the deformation thresholds of RF patterns because regardless of the RF or radius, the radial deformation thresholds are closely matched across the entire range of circular contour frequencies, suggesting that the patterns with the same circular contour frequency should have the same radial deformation threshold (Jeffrey et al., 2002).

To examine the dependence of the radial frequency discrimination thresholds on the radius (eccentricity), we used the RF reference patterns with constant CCF $\left(\mathrm{r}_{0} / \omega=\right.$ constant) and constant absolute modulation amplitude $\left(\mathrm{Ar}_{0}=\right.$ constant $)$ for changes in radius $(4 \sim 16 \mathrm{deg})$. The constant CCF RF patterns were created by covarying the radius and radial frequency such that they have an identical CCF regardless of the change in the radius. Figure $3 \mathrm{a}$ shows a schematic of an example of the constant CCF RF pair. The two patterns with different radii and RFs have the same CCF. Moreover, the $\sigma$ of the two patterns remains constant. These two patterns' cycles of modulation travel the same length in physical space. We used three series of constant $\mathrm{CCF}(\mathrm{CCF}=0.358$ cycles $/$ cl-degree $)$ patterns with different radii $(4,8,12$, and $16 \mathrm{deg})$ but the same thickness $(\sigma=1 \mathrm{deg})$ as the reference stimuli. Figure $3 \mathrm{~b}$ shows the experimental reference stimuli used in this experiment.

The absolute modulations $\left(\mathrm{r}_{0} \mathrm{~A}\right)$ of the reference RF patterns were held constant $(0.12,0.24,0.48 \mathrm{deg})$ within a session but were different across sessions. The product of $\mathrm{r}_{0} \mathrm{~A}$ and the contour length reflects the size of a single cycle of the RF patterns. This indicates that the size of the stimuli was held constant within a session.

\section{Results}

In Experiment 1, the discrimination thresholds were measured as a function of the radius over a range from 4 to $16 \mathrm{deg}$ (14 deg instead of $16 \mathrm{deg}$ when $\mathrm{r}_{0} \mathrm{~A}=0.12 \mathrm{deg}$, because of the impossibility of measurement otherwise) for the five participants. In Fig. 4, the mean of the discrimination thresholds for the five participants is plotted against the radius. The thresholds vs. radius functions were strongly dependent on the stimulus conditions $\left(\mathrm{r}_{0} \mathrm{~A}\right)$ of the target and reference stimuli. The thresholds substantially increased with increasing radius for the condition $\mathrm{r}_{0} \mathrm{~A}=$ $0.12 \mathrm{deg}$, i.e., the small stimulus size and slightly increased for the condition $\mathrm{r}_{0} \mathrm{~A}=0.24 \mathrm{deg}$, while they remained constant for the condition $\mathrm{r}_{0} \mathrm{~A}=0.48$ deg, i.e., the large stimulus size. In this instance, the increase in the threshold implied a decrease in the discrimination performance, which indicates a radius effect of the RF patterns. The radius effect was observed for the condition $\mathrm{r}_{0} \mathrm{~A}=0.12$ deg, but not for the condition $\mathrm{r}_{0} \mathrm{~A}=0.48 \mathrm{deg}$. To test for significant differences in the group data, we used three one-way ANOVA. Based on the results, a main effect for the condition $\mathrm{r}_{0} \mathrm{~A}=0.12 \mathrm{deg}, F(3,12)=9.627, p<0.05$; 


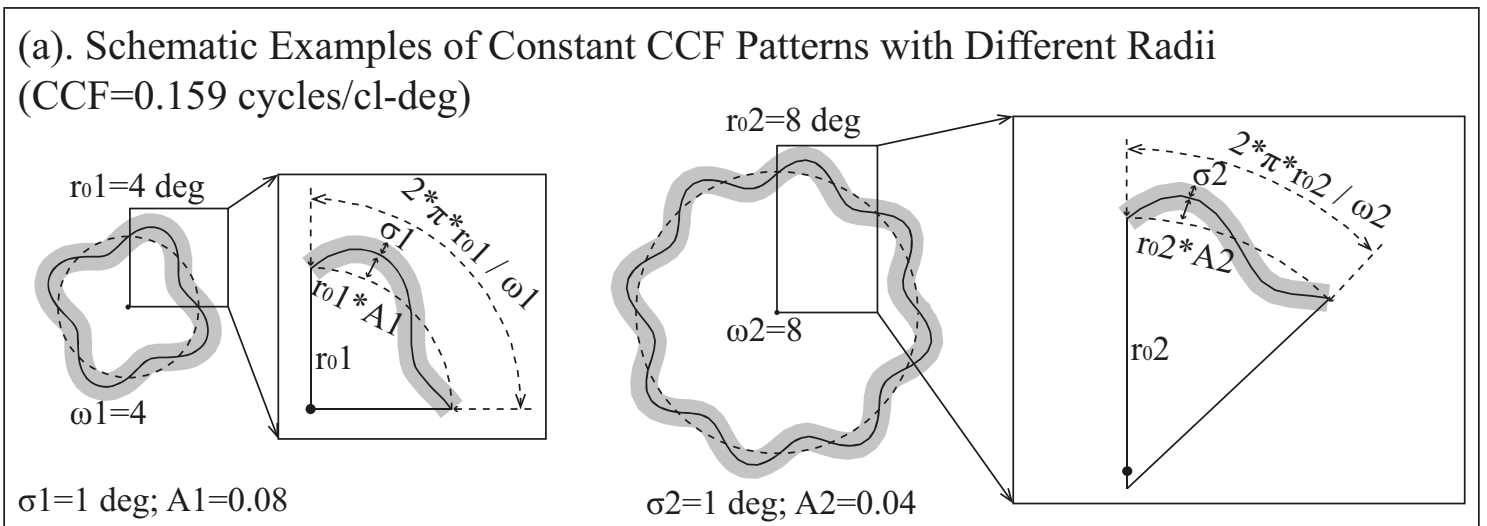

(b). Constant CCF Patterns with Different Radii Used in Experiment 1 $(\mathrm{CCF}=0.358$ cycles/cl-deg; $\sigma=1 \mathrm{deg})$

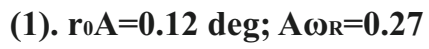

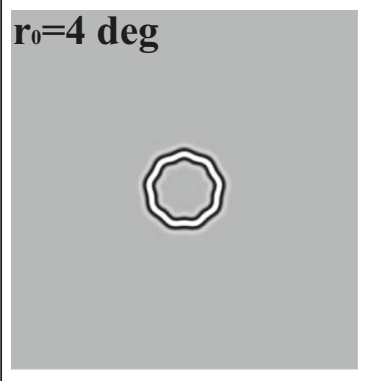

$\omega_{\mathrm{R}}=9 \mathrm{cycles} / 360 \mathrm{deg}$

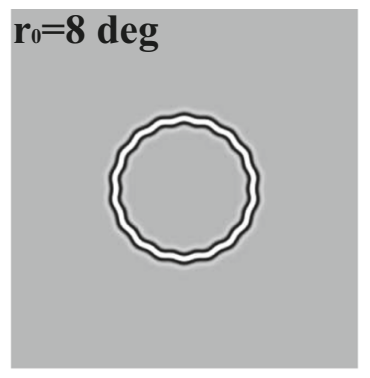

$\omega_{\mathrm{R}}=18 \mathrm{cycles} / 360 \mathrm{deg}$

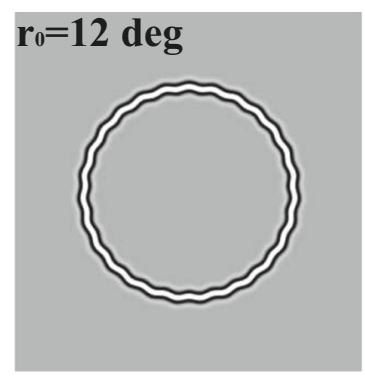

$\omega_{\mathrm{R}}=27 \mathrm{cycles} / 360 \mathrm{deg}$

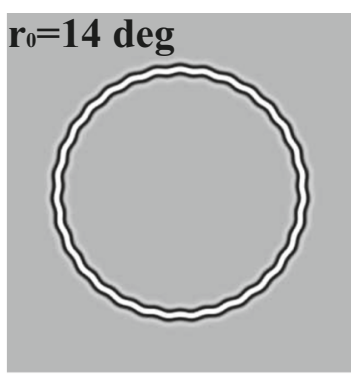

$\omega_{\mathrm{R}}=31 \mathrm{cycles} / 360 \mathrm{deg}$

(2). $\mathrm{r}_{0} \mathrm{~A}=0.24 \mathrm{deg} ; \mathrm{A} \omega_{\mathrm{R}}=0.54$

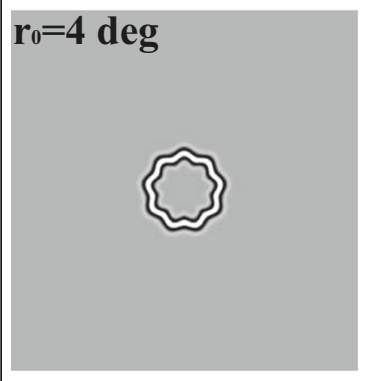

$\omega_{\mathrm{R}}=9 \mathrm{cycles} / 360 \mathrm{deg}$

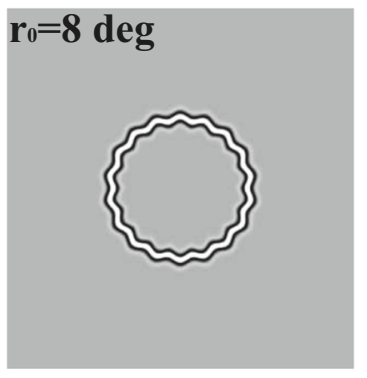

$\omega_{\mathrm{R}}=18 \mathrm{cycles} / 360 \mathrm{deg}$

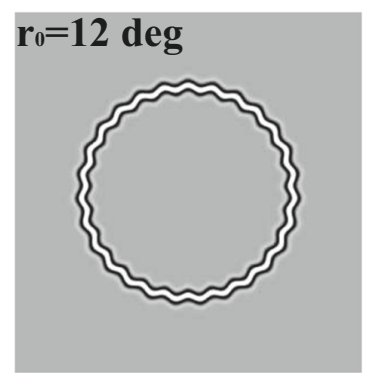

$\omega_{\mathrm{R}}=27 \mathrm{cycles} / 360 \mathrm{deg}$

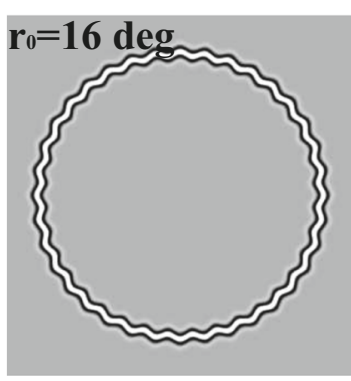

$\omega_{\mathrm{R}}=36 \mathrm{cycles} / 360 \mathrm{deg}$

(3). $\mathrm{r}_{0} \mathrm{~A}=0.48 \mathrm{deg} ; \mathrm{A} \omega_{\mathrm{R}}=0.1 .08$

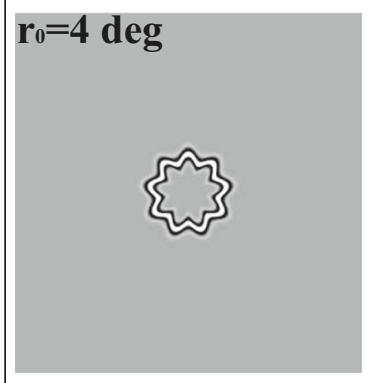

$\omega_{\mathrm{R}}=9 \mathrm{cycles} / 360 \mathrm{deg}$

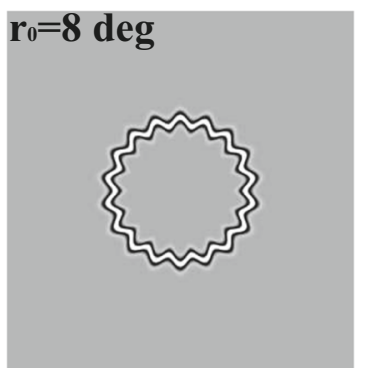

$\omega_{\mathrm{R}}=18 \mathrm{cycles} / 360 \mathrm{deg}$

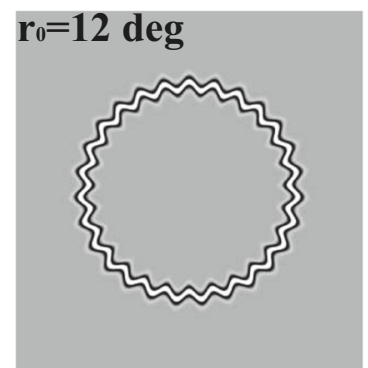

$\omega_{\mathrm{R}}=27 \mathrm{cycles} / 360 \mathrm{deg}$

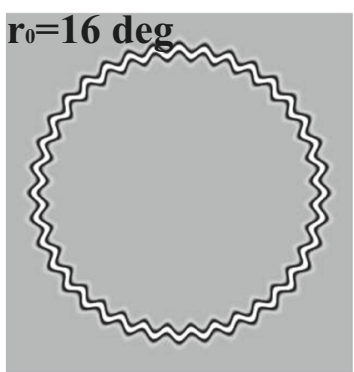

$\omega_{\mathrm{R}}=36 \mathrm{cycles} / 360 \mathrm{deg}$ and the condition $\mathrm{r}_{0} \mathrm{~A}=0.24 \mathrm{deg}, F(3,12)=23.606, p<$ 0.05 has been found. However, no main effect for the condition $\mathrm{r}_{0} \mathrm{~A}=0.48 \mathrm{deg}, F(3,12)=0.197, p=0.77$ has been found. 
Fig. 3 Constant circular contour frequency $(\mathrm{CCF})$ patterns. To examine the dependence of radial frequency discrimination thresholds on the radius (eccentricity), we use a series of CCF constant patterns with different radii $(4,8,12$, and $16 \mathrm{deg})$. The $\mathrm{CCF}$ is considered to be an important factor in the visual performance of RF patterns regardless of radial frequency or radius (see text). (a) shows schematic examples explaining how two patterns with different radii $\left(\mathrm{r}_{0}\right)$ and radial frequencies $(\omega)$ have the same circular contour frequency $(\mathrm{CCF}=0.159$ cycles/cl-deg). $\mathrm{CCF}$ is defined by the number of radial cycles per deg of unmodulated contour length measured in deg of viewing angle and calculated by $\omega / 2 \pi \mathrm{r}_{0}$. (b) shows three series of reference patterns with a constant CCF of 0.358 cycles/cl-deg and a constant MOD $\left(\mathrm{A} \omega_{\mathrm{R}}\right)$ used to examine the dependence of the pattern radius in experiment 1 . Here, MOD is the Maximum orientation difference between the RF pattern and the unmodulated circle. The reference radial frequency $\left(\omega_{R}\right)$ increases with increasing radius to keep the CCF constant $(0.358$ cycles/cl-deg $)$; meanwhile, the $\sigma$ of the patterns remains constant $(\sigma=1 \mathrm{deg})$. The absolute modulations $\left(\mathrm{r}_{0} \mathrm{~A}\right)$ of the target and the reference RF patterns hold constant $(0.12,0.24$, $0.48 \mathrm{deg}$ as shown in (1), (2) and (3)) within a session but are different across sessions. Also, MODs of the reference stimuli holds constant $\left(\mathrm{A} \omega_{\mathrm{R}}=0.27,0.54,1.08\right)$ within a session. We use seven radial frequencies for the target stimuli $\left(\omega_{\mathrm{T}}\right)$ : the same frequency as the reference, and three higher and three lower than that of the reference. We use 14 deg instead of 16 deg when $r_{0} \mathrm{~A}=0.12$ deg because of the impossibility of measurement otherwise. Meanwhile, we use 31 cycles $/ 360 \mathrm{deg}$ as the reference stimulus to keep the CCF constant. Note here that four constant $\mathrm{CCF}$ reference patterns with different radii within a session have the same orientation difference between RFs and a circle at crossing point

\section{Discussion}

As shown in Fig. 4, the discrimination thresholds between different-frequency RF patterns vs. radius functions for the constant CCF RF patterns indicated different functional forms dependent on the modulation amplitude of the RF patterns. The thresholds showed an increasing functional form for small modulation amplitude RF patterns $\left(\mathrm{r}_{0} \mathrm{~A}=0.12\right.$ and $\left.0.24 \mathrm{deg}\right)$ but a flattened function for large amplitude RF patterns $\left(\mathrm{r}_{0} \mathrm{~A}=\right.$ $0.48 \mathrm{deg})$.

\section{The effect of the pattern contrast (100\%)}

We used the pattern contrast of $100 \%$ through all present experiments. The relationship between the physical variables and radial frequency discriminations does not always involve a simple one-to-one mapping between the two variables. The other stimulus characteristics which are likely to modify the relationship include the contrast and spatial frequency of the base stimulus. We used the stimulus contrast of $100 \%$ and the peak spatial frequency of $0.45 \mathrm{c} / \mathrm{deg}$ in the Experiment 1 . The perceived contrast of the Gabor patches decreases with increasing eccentricity $(0 \sim 13 \mathrm{deg})$ even partially compensating by scaling stimulus size in the periphery (Vanston and Crognale, 2018). The shape discrimination threshold of RF pattern of 4 cycles $/ 360$ deg from a circle decreases with increasing stimulus contrast from about $20 \sim 100 \%$ for the condition of spatial frequency $=1 \mathrm{cpd}$ and radius $=2.5 \mathrm{deg}$,

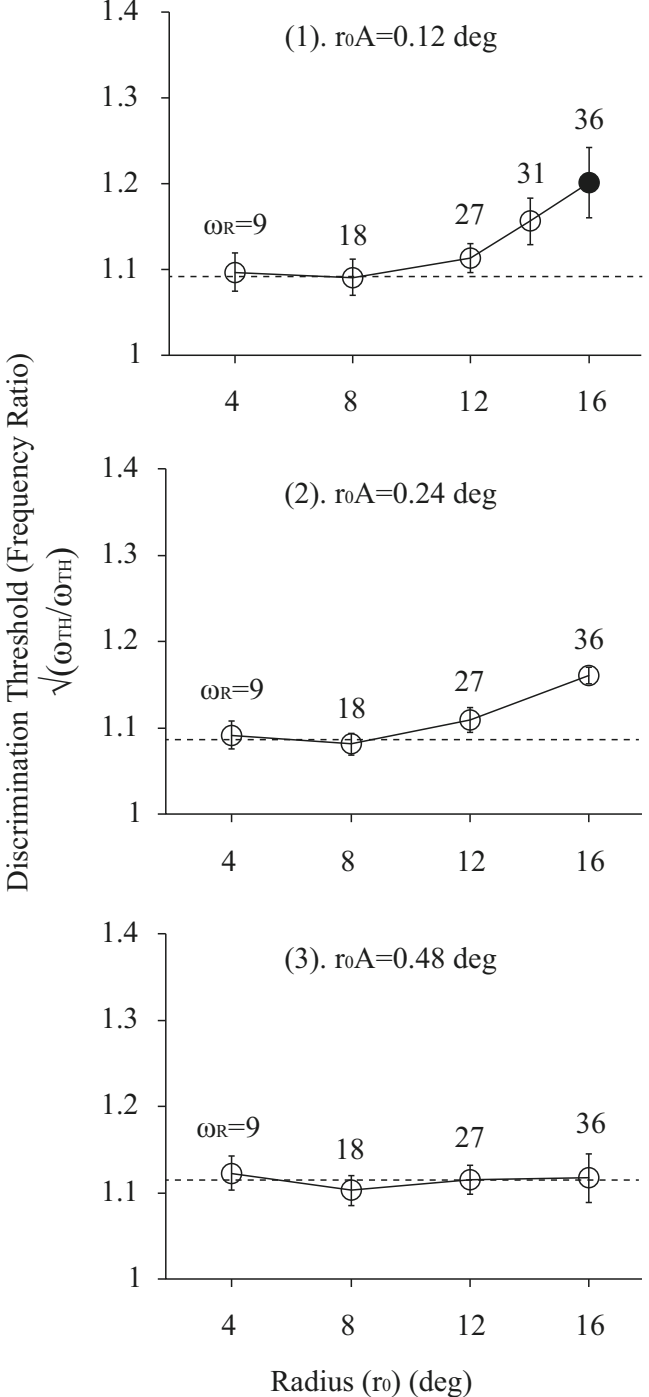

Fig. 4 Results of radial frequency discrimination for Experiment 1. Mean discrimination thresholds for the five participants plotted against the radius $(4,8,12$, and $16 \mathrm{deg})$. Thresholds (frequency ratio) are expressed as the square root of the higher frequency/lower frequency on the ordinate. The absolute amplitude $\left(r_{0} \mathrm{~A}\right)$ was held constant in each session but was varied across sessions; discrimination thresholds are shown for the small stimulus size: $0.12 \mathrm{deg}(1)$, the middle stimulus size: $0.24 \mathrm{deg}(2)$, and the large stimulus size: $0.48 \mathrm{deg}(3)$. When $\mathrm{r}_{0} \mathrm{~A}=0.12 \mathrm{deg}$, the threshold for $16 \mathrm{deg}$ is estimated from the thresholds for 12 and $14 \mathrm{deg}$ that were measured in the experiment. Error bars $=1$ S.E.

but not for the condition of spatial frequency $5 \sim 10 \mathrm{cpd}$ and radius $=2.5$ or $1 \mathrm{deg}$ ) (Ivanov and Mullen, 2012). The shape discrimination threshold of RF pattern decreases with increasing stimulus contrast from $12.5 \sim 100 \%$ for the condition of radial frequency of $5 \sim 12$ cycles, but not for the condition of redial frequency of $2 \sim 4$ cycles $/ 360 \mathrm{deg}$ (Wilkinson et al., 1998). This implies that the reduction in the perceived contrast with increasing eccentricity may result in increase of discrimination thresholds observed at eccentricity of $16 \mathrm{deg}$ for the condition of $\mathrm{r}_{0} \mathrm{~A}=0.12$ and $0.24 \mathrm{deg}$ in Fig. 4. However, only the reduction in the perceived contrast with increasing 
eccentricity cannot explain our result indicating the constant discrimination thresholds for the condition of $\mathrm{r}_{0} \mathrm{~A}=0.48 \mathrm{deg}$ regardless of eccentricity change.

\section{Pattern radius effects of radial frequency discrimination thresholds for constant CCF RF patterns}

The path of the RF patterns is less salient as the radius of the RF pattern increases. It might be the case that for the patterns with $\mathrm{r}_{0} \mathrm{~A}=0.12$ (and to a lesser extent 0.24 ) the larger receptive fields (lower spatial frequency preference) span cycles of modulation and smooth the percept of the path. This might result in an increase in the threshold.

It is well established that the performance of visual discrimination, such as the frequency, orientation, temporal frequency, vernier acuity, blur, displacement, and separation, is dependent on the signal strength, such as the contrast and size of a stimulus, with performance improving as the signal increases (Bowne, 1990; Reisbeck and Gegenfurtner, 1998; Skottun et al., 1987; Webster et al., 1990; Westheimer et al., 1999; Marechal and Shapley, 2004).

If radial frequency discrimination is processed by the orientation selective mechanism, our results may show common behavior with previous studies concerning orientation discrimination performance.

Specifically, in experiments where the stimuli (grating, Gabor element) were small, increasing the contrast lowered the orientation threshold (Regan and Beverley, 1985; Skottun et al. 1987; Reisbeck and Gegenfurtner, 1998; Mcllhagga and Mullen, 1996), whereas in the experiments when the stimuli were large, orientation discrimination thresholds were fairly contrast independent (Bowne, 1990; Skottun et al., 1987).

In the experiments performed by manipulating stimulus size, threshold estimates were lowest (acuity highest) for the largest size patch and increased as the stimulus size was reduced (Henrie and Shapley, 2001); the differential thresholds or JNDs in the orientation of a line stimulus at a given reference orientation (horizontal and oblique) decreased up to a stimulus length of $1 \mathrm{deg}$; over $1 \mathrm{deg}$, discrimination was not improved for the oblique orientation while for the horizontal orientation it improved gradually up to a length of $8 \mathrm{deg}$ (Orban et al., 1984). Mareschal and Shapley (2004), by varying systematically both contrast and size of a circular patch of grating, found that orientation discrimination thresholds decreased as contrast was increased for stimuli roughly smaller than $0.5 \sim 0.8 \mathrm{deg}$ in diameter, and as size was increased for stimuli with less than $4 \sim 20 \%$ grating contrast. The thresholds were saturated at constant level for larger stimuli than $1 \mathrm{deg}$ in diameter and with greater than $20 \%$ contrast.

The general finding was that as the stimulus signals (contrast and/or size) increased, the orientation discrimination thresholds for grating patches decreased rapidly at first, then gradually and finally asymptotically approached a saturated level.

We asked whether the above context concerning the signal strength effect on orientation discrimination can explain the results from Experiment 1.

We hypothesized that under strong signal strength (large modulation amplitude) conditions, frequency discrimination thresholds might not be as affected by the modulation amplitude and would remain constant at the saturation level whereas they would be increased under low signal strength (small modulation amplitude) condition.

To test this hypothesis, we estimated the perceptual strength of the stimuli in Experiment 1, which is considered to correspond to the signal strength of the stimuli such as the contrast of the grating stimuli in orientation discrimination, and examined the dependence of radial frequency discrimination on perceptual strength.

These estimations were carried out by dividing the stimulus modulation parameter $\mathrm{r}_{0} \mathrm{~A}$ of the RF pattern by the amplitude thresholds to detect the deformation of the base circles which were reported in our other study (Feng et al., 2020).

Table 1 lists the amplitude detection thresholds reported and the estimations represented as the multiples of the deformation threshold. The perceptual strength increases as the eccentricity decreases, from 1.8 to 7.3 for $\mathrm{r}_{0} \mathrm{~A}=$ 0.12 , from 3.5 to 14.5 for $\mathrm{r}_{0} \mathrm{~A}=0.24$ and from 7.1 to 29.1 for $\mathrm{r}_{0} \mathrm{~A}=0.48$. If the asymptote at which the signal strength effect saturates is approximately seven times the detection threshold, our hypothesis might suggest that above this level, the radial frequency discrimination thresholds may hold constant, whereas they may decrease with increasing signal strength up to the abovementioned asymptote.

The exception to this suggestion is the lack of a decreasing in threshold for the RF patterns when the radius decreases from 8 deg to 4 deg and the signal strength increases from 3.6 to 7.3 in the case of $r_{0} \mathrm{~A}=0.12 \mathrm{deg}$. However, the hypothetical suggestion is true in all the other conditions. It is not unreasonable that the radius size effect of the radial frequency discrimination seen in experiment 1 can be well explained by the context concerning the signal strength effect on orientation discrimination.

As a result, our finding that the radial frequency discrimination thresholds as a function of radius were dependent on the modulation amplitudes of the RF patterns is ascribed to the eccentricity effect reported in our other study (Feng et al., 2020). wherein the deformation thresholds required to discriminate the RF pattern from a circle increased with increasing stimulus eccentricity. Our results showed similar properties to previous studies concerning orientation discrimination performances, suggesting that the local contour orientation may be a critical cue for discriminating different frequency RF patterns. 
Table 1 The deformation thresholds in our other study and the stimulus modulation amplitudes (A) in the present study

\begin{tabular}{lllll}
\hline Radius (deg) & Results of our other study (Feng, et.al. 2020) & \multicolumn{3}{l}{ Stimulus parameters for the radial frequency discrimination experiment } \\
\cline { 3 - 5 } & Amplitude & $\mathrm{r}_{0} \mathrm{~A}=0.12 \mathrm{deg}$ & $\mathrm{r}_{0} \mathrm{~A}=0.24 \mathrm{deg}$ & $\mathrm{r}_{0} \mathrm{~A}=0.48 \mathrm{deg}$ \\
\hline $\mathrm{r}_{0}$ & Threshold amplitude & Perceptual amplitude (A /threshold) & 29.1 \\
4 & 0.0041 & 7.3 & 7.5 & 14.4 \\
8 & 0.0042 & 3.6 & 4.9 & 9.8 \\
12 & 0.0041 & 2.5 & 3.5 & 7.1 \\
16 & 0.0042 & 1.8 & & \\
\hline
\end{tabular}

We mention here that the effect of number of cycles of modulation of RF patterns should be examined to investigate locality or globality (global integration) of information processing of RF pattern in the peripheral field.

Dickinson, McGinty, Webster, and Badcock (2012) showed that the detection thresholds from circle described by gradient at a zero crossing decrease with increasing the number of cycles of modulation, being coincident at each value for number of cycles of nodulation regardless of RF. In a future work, we will examine the effect of number of number of cycles of modulation on frequency discrimination threshold between different RF patterns.

\section{Experiment 2}

\section{Magnified RF pattern}

In Experiment 1, using a constant CCF RF pattern, the radial frequency discrimination thresholds as a function of radius were influenced by the eccentricity effect, wherein the deformation thresholds required to discriminate the RF pattern from a circle increased with increasing stimulus eccentricity. Experiment 2 was designed to test whether spatial scaling (the retinocortical transformation) can neutralize the eccentricity effect observed in Experiment 1.

There exist many magnification factors reported (Anstis, 1974; Farrell and Desmarais, 1990; Higgins et al., 1996; Levi et al., 1985; Rovamo and Virsu, 1979; Melmoth and Rovamo, 2003; Schwartz, 1977; Watson, 1987; Whitaker et al., 1992). In the present experiment, we used Schwartz's retinocortical mapping function.

We constructed a series of magnified stimuli with different radii that had the same form in the V1 field and examined the dependence of the radial frequency discrimination thresholds for the magnified patterns on stimulus radius.

The magnified RF patterns with the CCF being inversely proportional to the radius of the stimuli, were created using Schwartz's retinocortical mapping function. Schwartz showed that the two-dimensional retinocortical mapping functions are represented in complex variables as in Eq. (5) (Schwartz, 1977):
$\mathrm{W}(\mathrm{Z})=\mathrm{k} \times \log (\mathrm{Z}+\mathrm{a})$

where the complex variable $\mathrm{Z}$ represents a point in the retinal plane; the complex variable $\mathrm{W}$ represents a point on the cortical plane; a defines the extent of the foveal singularity; and $\mathrm{k}$ is a scaling constant. For the value of a, there is rough consensus that the primate visual cortex is, to a first approximation, a complex logarithmic structure characterized by a foveal constant ranging from $\mathrm{a}=0.3 \mathrm{deg}$ to $\mathrm{a}=0.9 \mathrm{deg}$ (Wilson et al., 1990). In this study, we used $\mathrm{a}=0$ for the retina cortex function because our experimental condition was focused on $\mathrm{z} \geq 4 \mathrm{deg}$.

If one changes $r_{0}$ and $\sigma$ in proportion to the changing eccentricity while maintaining A and constant, then the changed patterns have a similar form; however, their radii are different and are dependent on eccentricity in a linear manner. If these changed patterns, as shown in Fig. 5a (the magnified RF patterns), are transformed from the retinal plane into the cortical plane using Eq. (6), the transformed patterns are different in position in the cortical plane; however, they have the same forms and radii, which implies that all transformed patterns activate the cortical area equally.

In this experiment, we used the magnified RF patterns with an identical reference RF. The reference RFs ( 9 and 18 cycles/ $360 \mathrm{deg}$ ) were held constant within a session but were different across sessions. The amplitude modulations (A) of the target and reference RF patterns were held constant within a session but were different across sessions (0.03 and 0.06$)$, as shown in Fig. 5 (b).

\section{Results}

For the magnified patterns, the thresholds were measured as a function of the pattern radius over a range from 4 to $16 \mathrm{deg}$. In Fig. 6, the means of the thresholds for the five participants are plotted against the radius under the four conditions $\left(\omega_{R}=9\right.$ cycles $/ 360$ deg and $\mathrm{A}=0.03 ; \omega_{\mathrm{R}}=9$ cycles $/ 360 \mathrm{deg}$ and $\mathrm{A}=$ $0.06 ; \omega_{R}=18$ cycles $/ 360$ deg and $A=0.03 ;$ and $\omega_{R}=18$ cycles $/ 360$ deg and $A=0.06$ ). As shown in Fig. 6 , the thresholds remained constant across all radii, approximately 1.1 , and regardless of the other stimulus parameters. To test for significant differences in the group data, we used a three-way 
(a). Schematic Examples of Magnified Patterns with Different Radii

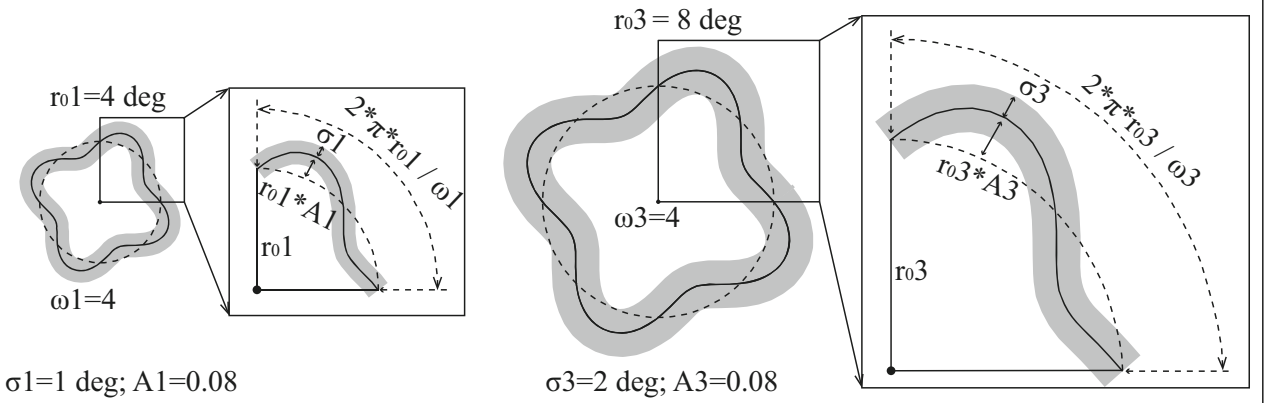

(b). Magnified Patterns with Different Radii Used in Experiment 2

(1). $\omega_{\mathrm{R}}=9$ cycles $/ 360 \mathrm{deg} ; \mathrm{A}=0.03 ; ; A \omega_{\mathrm{R}}=0.27$
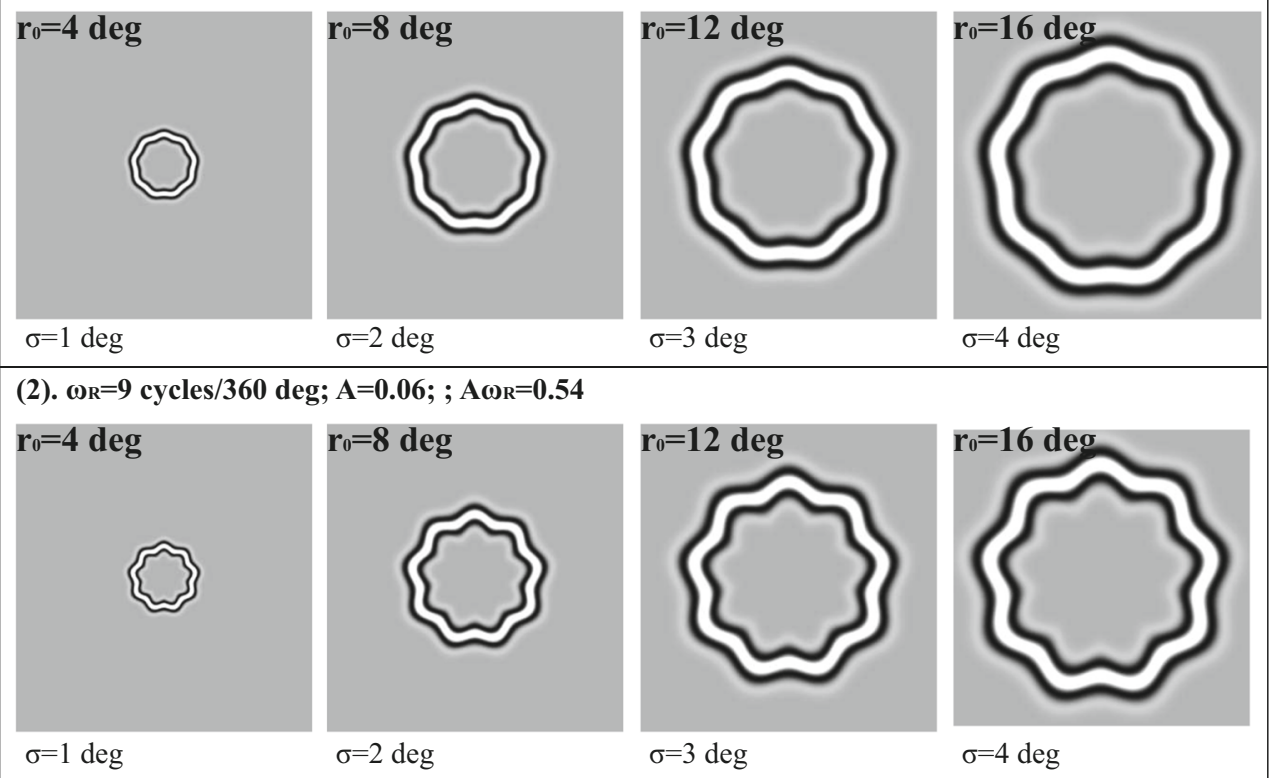

(3). $\omega_{\mathrm{R}}=18 \mathrm{cycles} / 360 \mathrm{deg} ; \mathrm{A}=0.03 ; ; A \omega_{\mathrm{R}}=0.54$
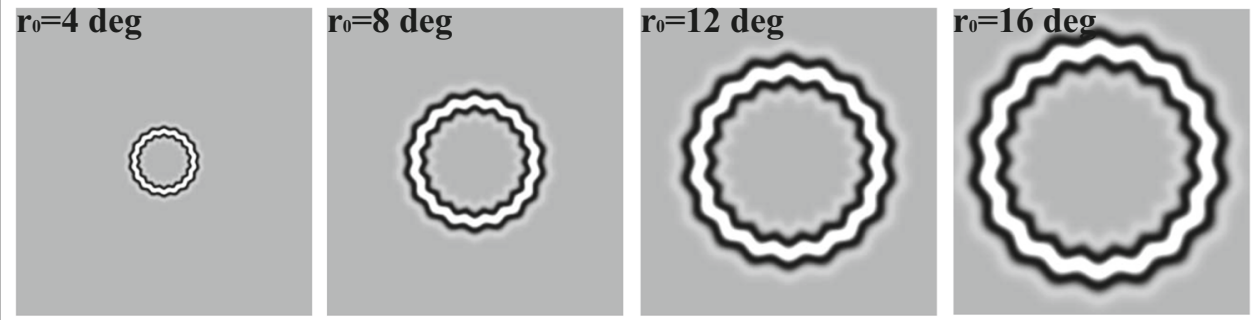

$\sigma=1 \mathrm{deg}$

$\sigma=2 \operatorname{deg}$

$\sigma=3 \mathrm{deg}$

$\sigma=4 \operatorname{deg}$

(4). $\omega_{\mathrm{R}}=18$ cycles $/ 360 \mathrm{deg} ; A=0.06 ; ; A \omega_{\mathrm{R}}=1.08$
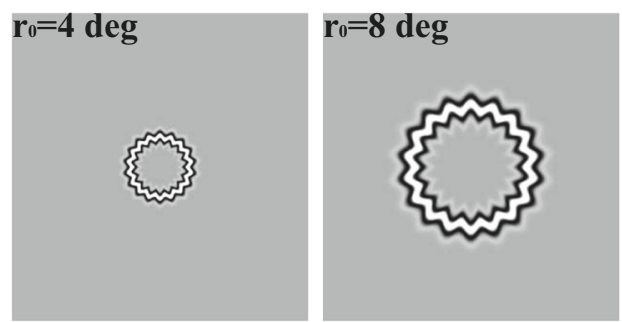

$\sigma=1 \operatorname{deg}$

$\sigma=2 \operatorname{deg}$

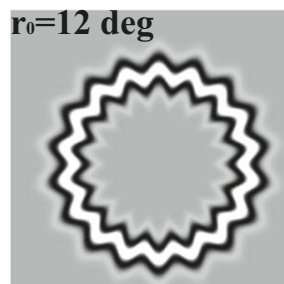

$\sigma=3 \mathrm{deg}$

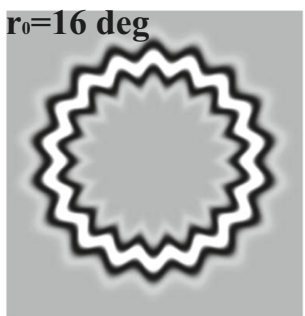

$\sigma=4 \operatorname{deg}$ 
Fig. 5 Magnified patterns in Experiment 2. (a) shows schematic examples created by using Schwartz's retinocortical mapping function. These patterns are different in position in the cortical plane but have the same forms and radii, implying that all the transformed patterns activate the cortical area equally. (b) shows the four series of reference magnified patterns used in Experiment 2. $\sigma$ increases with increasing radius, while the radial frequency $\left(\omega_{R}\right)$ and amplitude are constant $\left(\omega_{R}=9\right.$ cycles $/ 360$ deg, $A=0.03, \omega_{R}=9$ cycles $/ 360 \mathrm{deg}, A=0.06, \omega_{R}=18$ cycles $/ 360 \mathrm{deg}$, $\mathrm{A}=0.03$ and $\omega_{\mathrm{R}}=18$ cycles $/ 360 \mathrm{deg}, \mathrm{A}=0.06$ as shown in (1), (2), (3) and (4)) within a session but are different across sessions. Note here that four magnified reference patterns with different radii within a session have the same orientation difference between RFs and a circle at crossing. Also, MODs of the reference stimuli holds constant $\left(A \omega_{R}=0.27,0.54\right.$, $0.54,1.08$ ) within a session but are different across sessions

ANOVA. ANOVA with the factors of radius, A and $\omega_{R}$, was used to test for main effects. Based on the results, no main effect for the three factors: radius, $F(3,12)=4.771, p=0.086$; $\mathrm{A}, F(1,4)=0.25, p=0.643$; and $\omega_{\mathrm{R}}, F(1,4)=0.019, p=$ 0.896 has been found.

The radial frequency discriminability of the target stimuli from the reference stimulus is represented by the difference between the response frequencies corresponding to the "higher" responses and the "lower" responses. Figure 7 shows the cumulative discriminability of the target from the reference stimuli versus the target radial frequency over all participants and the four radii for each magnified pattern $\left(\omega_{R}=9\right.$ cycles $/ 360 \mathrm{deg}$ and $\mathrm{A}=0.03 ; \omega_{\mathrm{R}}=9 \operatorname{cycles} / 360 \mathrm{deg}$ and $\mathrm{A}=$ $0.06 ; \omega_{R}=18$ cycles $/ 360 \mathrm{deg}$ and $A=0.03$; and $\omega_{R}=18$ cycles/360 deg and $A=0.06$ ). A positive value on the ordinates indicates the magnitude of discrimination performance for a target that the participants judge to have a higher frequency than the reference frequency (9 cycles/360 deg), whereas a negative value indicates the magnitude of discrimination performance for a target that the participants judge to have lower radial frequency than the reference frequency. Discriminability increases as the target radial frequency increases (or decreases) from the reference frequency of 9 cycles/360 deg. The discriminability function shows a linear function of target frequency on the logarithmic scale within the frequency domain being presented.

\section{Discussion}

\section{Comparison of our discrimination thresholds with the identification accuracies in previous studies}

Wilkinson et al. (1998) found that participants were able to identify RF patterns of six or less cycles with an accuracy of over $90 \%$ and seven or more cycles with an accuracy of $60 \%$ or less. These identification accuracies were also observed by Poirier and Wilson (2006). The present study shows that the radial frequency discrimination thresholds (frequency ratio) are approximately 1.1 for RF patterns with a large stimulus size $\left(\mathrm{r}_{0} \mathrm{~A}=0.48\right)$ and for the magnified stimuli, as shown in Fig. 4 (3) and Fig. 6, respectively.

Although these results cannot be compared with the identification accuracies in studies by Wilkinson et al. (1998) and Poirier and Wilson (2006) because of the different stimulus conditions, the logarithms of the ratios of 5 cycles to 6 cycles $(6 / 5=1.2)$ and 6 cycles to 7 cycles $(7 / 6=1.16)$ are larger than our radial frequency discrimination thresholds. This finding suggests that 5-cycle (or 6-cycle) RF patterns are easily

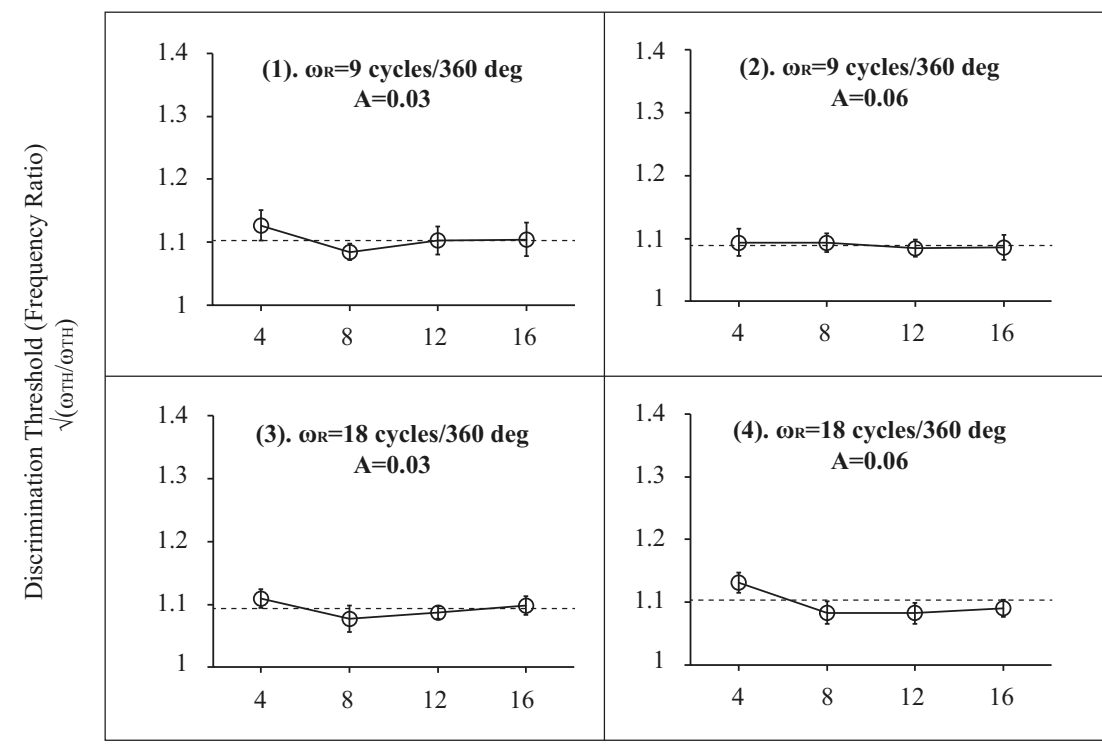

Radius (ro) (deg)

Fig. 6 Results of radial frequency discrimination Experiment 2. Mean discrimination thresholds for the five participants plotted against the radius $(4,8,12$, and $16 \mathrm{deg})$. Thresholds (frequency ratio) are expressed on the ordinate. The amplitude (A) and the radial frequency of the reference stimulus $\left(\omega_{R}\right)$ were held constant within a session but varied across sessions: $A=0.03 ; \omega_{R}=9$ cycles $/ 360 \operatorname{deg}(1), A=0.03 ; \omega_{R}=18$ cycles $/ 360 \operatorname{deg}(2), \mathrm{A}=0.06 ; \omega_{\mathrm{R}}=9 \operatorname{cycles} / 360 \operatorname{deg}(3)$, and $\mathrm{A}=0.03$; $\omega_{\mathrm{R}}=9$ cycles $/ 360 \mathrm{deg}$ (4). Error bars $=1$ S.E. 


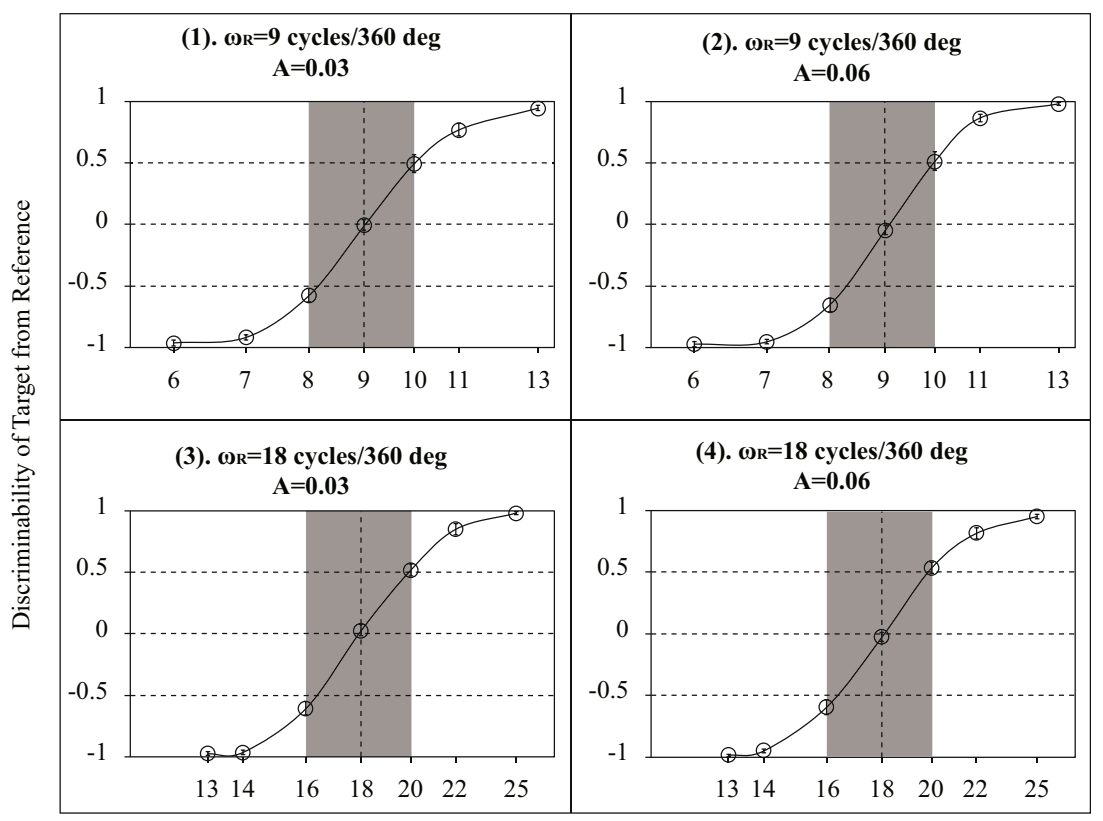

Target Radial Frequency (cycles/360 deg)

Fig. 7 Discriminability of the target from reference stimuli versus target radial frequency. The responses of the participants consist of their "higher", "lower", or "same" responses. The difference between the response frequencies of the "higher" responses and the "lower" responses is considered to represent the radial frequency discriminability of the target stimuli from the reference stimuli. Each panel shows the cumulative difference between the response frequencies of the "higher" responses and the "lower" responses over all participants and the four radii for each magnified pattern $\left(\omega_{R}=9\right.$

discriminated from 6-cycle (or 7-cycle) RF patterns. The results obtained for the RF patterns in this study are not inconsistent with the identification accuracies from Wilkinson et al. (1998) and Poirier and Wilson (2006).

\section{Frequency ratio determines the radial frequency discrimination thresholds}

Previous findings of radial frequency-selective effects, such as masking effects (Habak et al., 2006; Bell et al., 2007), adaptation effects (Bell et al., 2008; Bell et al., 2009), and subthreshold summation effects (Bell and Badcock, 2009), suggest that detection of the RF pattern may be processed by several RF channels, each channel selectively sensitive to a limited band of radial frequencies. These channels may also be involved in the suprathreshold perception of radial frequency and of the waveforms of RF patterns. If this is so, the ability to discriminate between different radial frequency patterns as measured in the present study might be determined by the activity difference of the multiple channels for the different patterns.

Figure 6 indicates that the radial frequency discrimination thresholds for the magnified RF stimuli were the same for two different reference frequencies (9 and 18 cycles/360 deg), cycles $/ 360 \mathrm{deg}, \mathrm{A}=0.03 ; \omega_{\mathrm{R}}=9$ cycles $/ 360 \mathrm{deg}, \mathrm{A}=0.06 ; \omega_{\mathrm{R}}=18$ cycles $/ 360 \mathrm{deg}, \mathrm{A}=0.03$; or $\omega_{\mathrm{R}}=18$ cycles $/ 360 \mathrm{deg}, \mathrm{A}=0.06$ ). Error bars $=1$ S.E. The observed small error bars imply little 1 variance across the participants and radii. Discriminability increases as the target radial frequency increases (or decreases) from the reference frequency of 9 cycles/360 deg. The discriminability function shows a linear function of target frequency on the logarithmic scale within the frequency domain being presented

implying that the ability to discriminate between the two different stimuli depends primarily on the ratio of their radial frequencies over a wide range of absolute radial frequencies. That we found equally discriminable radial frequencies to differ by nearly the same ratio may suggest that the spacing and bandwidth of the processing units of RF patterns may be more uniform if expressed on a logarithmic rather than a linear scale.

It should be mentioned here that the spatial frequency bandwidths of cortical cells turned to different spatial ranges have bandwidths that are roughly constant on a ratio, or logarithmic, scale (De Valois and De Valois 1990). Thus, equal logarithmic channel bandwidths have been reported for spatial frequency selectivity.

Our experimental results that the radial frequency discrimination thresholds were the same for the two different reference frequencies ( 9 and 18 cycles/360 deg) may be explain by a multiple channels model with equal logarithmic channel bandwidths of RF.

\section{Conclusions}

We found that the discrimination thresholds expressed by the frequency ratio between RF patterns of different frequencies 
vs radius functions for the constant CCF RF patterns indicated different functional forms dependent on the modulation amplitude of the RF patterns. This dependence was ascribed to the eccentricity effect wherein the deformation thresholds for discriminating the RF pattern from a circle increase with increasing stimulus eccentricity (Feng, et al., 2020).

We also found that the discrimination thresholds vs. radius functions for the magnified RF patterns were flattened for different modulation amplitudes and frequencies. The thresholds were similar at all eccentricities. Cortical magnification neutralized the eccentricity effect observed for the constant CCF patterns.

Acknowledgements The author(s) disclosed receipt of the following financial support for the research, authorship, and/or publication of this article: This study was supported by the Japan Society for the Promotion of Science KAKENHI (grant numbers 17K18855, $18 \mathrm{H} 05009,18 \mathrm{~K} 12149,18 \mathrm{~K} 8835,18 \mathrm{H} 01411,19 \mathrm{KK} 0099$ and 20K04381), National Natural Science Foundation of China (31700939), and a Grant-in-Aid for Strategic Research Promotion from Okayama University.

\section{References}

Achtman, R.L., Hess, R.F., and Wang, Y.Z. (2000). Regional sensitivity for shape discrimination. Spatial Vision 13, 377-391.

Anstis, S. M. (1974). A chart demonstrating variations in acuity with retinal position. Vision Research, 14, 589-592.

Bell, J., and Badcock, D.R. (2009). Narrow-band radial frequency shape channels revealed by sub-threshold summation. Vision Research 49, 843-850.

Bell, J., Badcock, D.R., Wilson, H., and Wilkinson, F. (2007). Detection of shape in radial frequency contours: independence of local and global form information. Vision Research 47, 1518-1522.

Bell, J., Dickinson, J.E., and Badcock, D.R. (2008). Radial frequency adaptation suggests polar-based coding of local shape cues. Vision Research 48, 2293-2301.

Bell, J., Wilkinson, F., Wilson, H.R., Loffler, G., and Badcock, D.R. (2009). Radial frequency adaptation reveals interacting contour shape channels. Vision Research 49, 2306-2317.

Bowne, S. F. (1990). Contrast discrimination cannot explain spatial frequency, orientation or temporal frequency discrimination. Vision Research, 30, 449-461.

Brainard, D.H. (1997). The Psychophysics Toolbox. Spatial Vision 10, 433-436.

De Valois, R.L., and De Valois K.K. (1990). Spatial Vision. Oxford University Press, Oxford

Dickinson, J.E., Bell, J., and Badcock, D.R. (2013). Near their thresholds for detection, shapes are discriminated by the angular separation of their corners. PLoS One 8, e66015.

Dickinson JE, McGinty J, Webster KE, and Badcock DR (2012) Further evidence that local cues to shape in RF patterns are integrated globally. Journal of Vision 12.

Farrell, J. E., and Desmarais, M. (1990). Equating character-identification performance across the visual field. Journal of the Optical Society of America, 7, 152-159.

Feng, Y., Wu, Q., Yang, J., Takahashi, S., Ejima, Y., Wu, J., and Zhang, M. (2020). Eccentricity Effect of Deformation Detection for Radial Frequency Patterns With Their Centers at Fixation Point. Perception, 49(8), 858-881.
García-Pérez, M.A. (2010), Denoising forced-choice detection data, The British Journal of Mathematical and Statistical Psychology, 63, 75100.

Habak, C., Wilkinson, F., and Wilson, H.R. (2006). Dynamics of shape interaction in human vision. Vision Research 46, 4305-4320.

Henrie, J. A., and Shapley, R. M. (2001). The relatively small decline in orientation acuity as stimulus size decreases. Vision Research, 41, $1723-1733$.

Higgins, K. E., Arditi, A., and Knoblauch, K. (1996). Detection and identification of mirror-image letter pairs in central and peripheral vision. Vision Research, 36, 331-337.

Ivanov, I.V., and Mullen, K.T., (2012) The role of local features in shape discrimination of contour- and surface-defined radial frequency patterns at low contrast. Vision Research. 52 1-10

Jeffrey, B.G., Wang, Y.Z., and Birch, E.E. (2002). Circular contour frequency in shape discrimination. Vision Research 42, 2773-2779.

Kaernbach, C. (2001). Adaptive threshold estimation with unforcedchoice tasks. Perception \& Psychophysics 63, 1377-1388.

Levi, D. M., Klein, S. A., and Aitsebaomo, A. P. (1985). Vernier acuity crowding and cortical magnification. Vision Research, 25, 963-977.

Loffler, G. (2008). Perception of contours and shapes: Low and intermediate stage mechanisms. Vision Research 48, 2106-2127.

Loffler, G. (2015). Probing intermediate stages of shape processing. Journal of Vision 15, 1.

Loffler, G., Wilson, H.R., and Wilkinson, F. (2003). Local and global contributions to shape discrimination. Vision Research 43, 519-530.

Mareschal, I., and Shapley, R.M. (2004). Effects of contrast and size on orientation discrimination. Vision Research 44, 57-67.

Mcllhagga, W. H., and Mullen, K. T. (1996). Contour integration with colour and luminance contrast. Vision Research, 9, 1265-1280.

Melmoth, D. R., and Rovamo, J.M. (2003), Scaling of letter size and contrast equalises perception across eccentricities and set sizes, Vision Research 43 (2003) 769-777.

Orban, G. A., Vandenbussche, E., and Vogels, R. (1984). Human orientation discrimination tested with long stimuli. Vision Research, 24, 121-128.

Pelli, D.G. (1997). The VideoToolbox software for visual psychophysics: transforming numbers into movies. Spatial Vision 10, 437-442.

Poirier, F.J., and Wilson, H.R. (2006). A biologically plausible model of human radial frequency perception. Vision Research 46, 2443 2455.

Regan, D., and Beverley, K. I. (1985). Post adaptation orientation discrimination. Journal of the Optical Society of America, 2, 147-155.

Reisbeck, T. E., and Gegenfurtner, K. R. (1998). Effects of contrast and temporal frequency on orientation discrimination for luminance and isoluminant stimuli. Vision Research, 38, 1105-1117.

Rovamo, J., and Virsu, V. (1979). An estimation and application of the human cortical magnification factor. Experimental Brain Research, 37, 495-510.

Salmela, V.R., Henriksson, L., and Vanni, S. (2016). Radial frequency analysis of contour shapes in the visual cortex. PLoS Computational Biology 12, e1004719.

Schmidtmann, G., and Fruend, I. (2019). Radial frequency patterns describe a small and perceptually distinct subset of all possible planar shapes. Vision Research 154, 122-130.

Schmidtmann, G. and Kingdom, F.A.A. (2017) Nothing more than a pair of curvatures: a common mechanism for the detection of both radial and non-radial frequency patterns. Vision Research 134, 18-25.

Schmidtmann, G., Logan, A.J., Kennedy, G.J., Gordon, G.E., and Loffler, G. (2015). Distinct lower visual field preference for object shape. Journal of Vision, 15 (5) (2015).

Schwartz, E.L. (1977). The development of specific visual connections in the monkey and the goldfish: outline of a geometric theory of receptotopic structure. Journal of Theoretical Biology 69, 655-683.

Skottun, B.C., Bradley, A., Sclar, G., Ohzawa, I., and Freeman, R.D. (1987). The effects of contrast on visual orientation and spatial 
frequency discrimination: a comparison of single cells and behaviour. Journal of Neurophysiology, 57, 773-786.

Vanston, J.E. and Crognale, M.A., (2018), Effects of eccentricity on color contrast. Journal of the Optical Society of America, Vol. 35, No. 4, B122-B129.

Watson, A. B. (1987). Estimation of local spatial scale. Journal of the Optical Society of America, 4, 1579-1582.

Webster, M. A., De Valois, K. K., and Switkes, E. (1990). Orientation and spatial-frequency discrimination for luminance and chromatic gratings. Journal of the Optical Society of America, 7, 1034-1049.

Westheimer, G., Brincat, S., and Wehrhahn, C. (1999). Contrast dependency of foveal spatial functions: orientation, vernier, blur and displacement discrimination and the tilt and Poggendorff illusions. Vision Research, 39, 1631-1639.

Whitaker D, Mäkela P, Rovamo J, and Latham K (1992). The influence of eccentricity on position and movement acuities as revealed by spatial scaling. Vision Research, 32,1913-1930.

Wilkinson, F., James, T.W., Wilson, H.R., Gati, J.S., Menon, R.S., and Goodale, M.A. (2000). An fMRI study of the selective activation of human extrastriate form vision areas by radial and concentric gratings. Current Biology 10, 1455-1458.
Wilkinson, F., Wilson, H.R., and Habak, C. (1998). Detection and recognition of radial frequency patterns. Vision Research 38, 35553568 .

Wilson, H.R., Levi, D., Maffei, L., Rovamo, J., and Devalois, R. (1990). The perception of form: Retina to striate cortex.

Zolubak, A. B., Garcia-Suarez, L. (2017) Regional sensitivity for shape discrimination (SD) in colour vision: concentric and eccentric presentations of radial frequency (RF) patterns. 40th European Conference on Visual Perception ECVP 2017

Zolubak, A. B., Garcia-Suarez, L. (2018) Eccentricity Effect in Shape Discrimination Hyperacuity Compensated by Scaling Factors Lower than the Cortical Magnification Factor. The Association for Research in Vision and Ophthalmology ARVO Annual Meeting 2018

Zolubak, A.B., Schmidtmann, G. \& Garcia-Suarez, L. (2018) Scaleinvariance for radial frequency patterns in peripheral vision. 41st European Conference on Visual Perception ECVP 2018

Publisher's note Springer Nature remains neutral with regard to jurisdictional claims in published maps and institutional affiliations. 